\title{
Utilization Pattern of Vancomycin in a University Teaching Hospital in Oman: Comparison with International Guidelines
}

\author{
Mohammed Al Za'abi ${ }^{1^{*}}$, Samreen Shafiq ${ }^{1}$, Dawood Al Riyami ${ }^{2}$ and Badreldin H \\ $\mathrm{Ali}^{\mathbf{1}}$ \\ ${ }^{1}$ Department of Pharmacology \& Clinical Pharmacy, ${ }^{2}$ Department of Medicine, College of Medicine \& Health Sciences, Sultan \\ Qaboos University, Muscat, Oman
}

*For correspondence: E-mail: zaabi@squ.edu.om; Tel: +96824141155; Fax: +96824141107

\begin{abstract}
Purpose: To assess the prescribing and utilization pattern of vancomycin in Sultan Qaboos University Hospital (SQUH) in Oman.

Methods: $A$ retrospective study that included in-patients at SQUH who had used vancomycin from January 12009 to December 312009 was conducted to determine the utilization patterns of the drug vis a vis to the Hospital Infection Control Practices Advisory Committee (HICPAC) guidelines and the North American Therapeutic Drug Monitoring Consensus (NATDMC) recommendations. Information regarding patient characteristics and vancomycin therapy were obtained from patients' medical records. Statistical analyses were performed using descriptive statistics.

Results: Out of 478 prescriptions for 365 patients, $79.1 \%$ were considered inappropriate, based on HICPAC guidelines. This was mainly due to the continuous use of vancomycin following negative microbiological cultures for $\beta$-lactam-resistant Gram-positive microorganisms. Vancomycin was prescribed mostly for treatment of sepsis (27.1\%), followed by prophylaxis against various clinical conditions (20.8\%). Therapeutic drug monitoring (TDM) practices at SQUH did not comply with NATDMC recommendations on the use of trough concentration in the specified range as a guide for monitoring of therapy.

Conclusion: Irrational use of vancomycin was recorded in this study. The need for a restrictive policy and a revision of the current TDM in the setting are recommended..
\end{abstract}

Keywords: Vancomycin, Drug utilization, Therapeutic drug monitoring, Infection

Tropical Journal of Pharmaceutical Research is indexed by Science Citation Index (SciSearch), Scopus, International Pharmaceutical Abstract, Chemical Abstracts, Embase, Index Copernicus, EBSCO, African Index Medicus, JournalSeek, Journal Citation Reports/Science Edition, Directory of Open Access Journals (DOAJ), African Journal Online, Bioline International, Open-J-Gate and Pharmacy Abstracts

\section{INTRODUCTION}

The glycopeptide antibiotic, vancomycin, is effective against most Gram-positive bacteria especially Staphylococcus aureus and methicillin-resistant Staphylococcus aureus (MRSA). Such efficacy favored wide-scale use of vancomycin which later resulted in increase in bacterial resistance. Since the discovery of the first strains of vancomycin-resistant bacteria in the late 1980s, the incidence of resistant strains has been rising worldwide [1]. Among these resistant strains were vancomycin-resistant enterococci (VRE) and vancomycin-intermediate Staphylococcus aureus (VISA) [2]. The emergence of these strains was attributed to excessive and inappropriate use of vancomycin [1-3]. The Hospital Infection Control Practices Advisory Committee (HICPAC), a subcommittee of the United States' Center for Disease Control 
and Prevention (CDC), released a protocol on the proper use of vancomycin and managing patients infected with resistant bacteria [4-5]. These recommendations became widely accepted in many hospitals worldwide.

Several studies evaluating the pattern of use of vancomycin have been conducted [6-8]. However, most of these were conducted in developed countries, and data on the utilization of vancomycin in developing regions remain sparse. Another significant limitation in many of these is the exclusion of TDM procedures recommended by the North American Therapeutic Drug Monitoring Consensus (NATDMC) from clinical practice [5]. . Vancomycin pharmacokinetics show large interand intra-individual variability for which serum drug assays are performed to ensure adequate therapy [5]. Proper use of TDM procedures along with appropriate prescription of vancomycin can help in preventing and controlling excessive use of vancomycin and the emergence of resistant microorganisms [9].

Thus, the purpose of this study was to evaluate the prescribing patterns and TDM of vancomycin at SQUH, a university teaching hospital in Oman, and to compare the results with the HICPAC recommendations and NATDMC.

\section{METHODS}

\section{Setting}

Sultan Qaboos University Hospital (SQUH) is a 518-bed tertiary-care hospital whose in-patient wards include four intensive care units (ICU), cardiac, oncology, neonatal, psychiatric, delivery, general medical, surgical and orthopedic wards. In addition, the hospital has bone marrow transplant and cardiothoracic units.

In SQUH, vancomycin is regarded as a restricted antibiotic. An antibiotic handbook published by SQUH in 2006 is used as primary protocol for utilization of vancomycin and it is available to every prescriber in the hospital (Table 1). The indications for the use of the drug are almost similar to those of HICPAC recommendations.

\section{Study design}

The study was carried out by reviewing all medical records of patients who were admitted and received vancomycin during a 12-month period, from January $1^{\text {st }} 2009$ to December $31^{\text {st }}$ 2009. Ethical approval was sought and obtained from Sultan Qaboos University Medical Ethics
Committee before commencing the study. Patients with missing clinical notes and/or no records of vancomycin utilization were excluded from the study.

Table 1: Recommended indications for the use of vancomycin in the antibiotic handbook of Sultan Qaboos University Hospital

\section{INDICATION}

- Methicillin resistant Staphylococcus aureus and methicillin-resistant Staphylcoccus epidermidis

- Serious enterococcal infections.

- Penicillin-resistant Streptococcus pneumonia (PRSP) infections .

- Clostridium difficile antibiotic-associated colitis.

- Central venous line infections caused by Staphylcoccus epidermidis and Staphylococcus aureus.

- Acute fulminant (post operative) endophthalmitis.

- Surgical prophylaxis in patients allergic to penicillin.

- An alternative in infective endocarditis caused by enterococci or Staphylococcus aureus.

- An alternative in cavernous sinus thrombophlebitis caused by Staphylococcus aureus.

A data collection form was used to gather patients' information. It included information about demographic data, history of drug allergy, co-morbidities, prescribing hospital unit, dosage regimen, co-medications, microbiological cultures and sensitivity testing, indication for use, application of TDM, clinical outcomes and reasons for discontinuation of therapy.

\section{Utilization and therapeutic drug monitoring studies}

Appropriate or inappropriate use of vancomycin was classified according to the guidelines issued by HICPAC. The TDM procedures applied in SQUH were compared to the TDM recommendations of NATDMC.

At SQUH, the serum concentrations of vancomycin were measured by fluorescence polarization immunoassay using semiautomatic analyzer (Roche Diagnostic Systems, USA). SQUH guidelines recommend a trough and a peak vancomycin serum concentration of $5-10$ $\mathrm{mg} / \mathrm{L}$ and $20-40 \mathrm{mg} / \mathrm{L}$, respectively. The sampling time for peak and trough vancomycin serum concentration was drawn $1 \mathrm{~h}$ postvancomycin infusion and right before the next dose, respectively. Vancomycin was given in a 
dose of $1000 \mathrm{mg}$ every $12 \mathrm{~h}$ for patients with normal renal function and in a dose of $1000 \mathrm{mg}$ every $24 \mathrm{~h}$ for patients with renal dysfunction.

\section{Statistical analysis}

SPSS version 16.0 (Chicago, IL, USA) was used to capture and analyze the data. Descriptive analysis was used to present the utilization of vancomycin and TDM procedures. Chi square $\left(x^{2}\right)$ and Fisher's exact tests were used to evaluate the association between gender, age and hospital unit and the appropriate prescribing of vancomycin based on HICPAC guidelines. Continuous variables such as age were analyzed by Student's t-test. $P$ values $<0.05$ were considered statistically significant.

\section{RESULTS}

\section{Patient population}

During the study period, vancomycin was prescribed 478 times to 365 patients. Of those patients, $53.2 \%$ were $>18$ years old with mean age and weight of $26.2 \pm 24.3$ years and $37.6 \pm$ $28.2 \mathrm{~kg}$, respectively. The male/female gender ratio was 1.1:1. The average dose of vancomycin given was $1066.1 \pm 691.1 \mathrm{mg}$ with an average duration of $7.2 \pm 6.9$ days. Almost all patients received vancomycin intravenously (98.5\%). Other clinical characteristics of the studied population are summarized in Table 2.

\section{Vancomycin utilization}

According to the HICPAC guidelines, vancomycin prescription was justified in only $20.9 \%(n=100)$ of the cases. There was discontinuation of the vancomycin therapy if microbiological cultures results were negative $(63.0 \%, n=63)$, treatment of infections caused by $\beta$-lactam resistant Gram-positive bacteria $(18.0 \%, n=18)$ and infections caused by Grampositive microorganisms in patients who are allergic to $\beta$-lactam antibiotics $(10.0 \%, n=10)$. Failure to discontinue vancomycin upon receiving reports with negative cultures for $\beta$-lactamresistant microorganisms accounted for most of the inappropriate prescriptions $(48.4 \%, \mathrm{n}=183)$. Other inappropriate uses of vancomycin were prophylactic use in patients requiring dialysis $(16.4 \%, \mathrm{n}=62)$, patients with indwelling catheters $(8.2 \%, \mathrm{n}=31)$ and for surgery in patients without $\beta$-lactam antibiotic allergies (2.9 $\%, n=11)$. There was no significant relationship between either demographic data or hospital unit and inappropriate prescribing of vancomycin.
Table 2: Clinical characteristics of patients receiving vancomycin at Sultan Qaboos University Hospital ( $\mathrm{n}=$ 365)

\begin{tabular}{|c|c|}
\hline \multirow{2}{*}{\multicolumn{2}{|c|}{ Hospital unit, n (\%) }} \\
\hline & \\
\hline Hematology & $122(33.4)$ \\
\hline Infectious diseases & $43(11.8)$ \\
\hline Nephrology & $43(11.8)$ \\
\hline Neurology & $29(7.9)$ \\
\hline Oncology & $28(7.7)$ \\
\hline Surgery & $24(6.6)$ \\
\hline Cardiology & $24(6.6)$ \\
\hline Others & $28(7.7)$ \\
\hline \multicolumn{2}{|l|}{ Dose $(\mathrm{mg})$, mean $( \pm S D)$} \\
\hline Prophylactic & $1069.1(451.7)$ \\
\hline Therapeutic & $1065.2(743.4)$ \\
\hline \multicolumn{2}{|l|}{$\begin{array}{l}\text { Treatment duration (days), mean } \\
( \pm S D)\end{array}$} \\
\hline Prophylactic & $2.2(2.5)$ \\
\hline Therapeutic & $8.5(7.2)$ \\
\hline \multicolumn{2}{|l|}{ Indication, n (\%) } \\
\hline Sepsis & $99(27.1)$ \\
\hline Prophylactic & $76(20.8)$ \\
\hline Other febrile conditions & $44(12.1)$ \\
\hline Pneumonia & $29(8.0)$ \\
\hline Meningitis & $15(4.1)$ \\
\hline Catheter-related infections & $31(8.5)$ \\
\hline Surgical prophylaxis & $13(3.6)$ \\
\hline $\begin{array}{l}\text { enterococci-related } \\
\text { infections }\end{array}$ & $3(0.8)$ \\
\hline $\begin{array}{l}\text { Acute chest syndrome } \\
\text { (sickle cell disease) }\end{array}$ & $7(2.0)$ \\
\hline Others & $48(13.2)$ \\
\hline \multicolumn{2}{|l|}{ Cultures results, $\mathrm{n}(\%)$} \\
\hline $\begin{array}{l}\text { Negative (no bacteria } \\
\text { identified) }\end{array}$ & $278(58.2)$ \\
\hline Gram positive cocci & $47(9.8)$ \\
\hline methicillin-sensitive & $29(6.1)$ \\
\hline Staphylococcus aureus & \\
\hline Coagulase negative & $24(5.0)$ \\
\hline Staphylococcus & \\
\hline methicillin-resistant & $18(3.8)$ \\
\hline Staphylococcus aureus & \\
\hline Others & $82(17.2)$ \\
\hline
\end{tabular}

\section{Therapeutic drug monitoring}

Of the recruited cases, only $7.4 \%(n=23)$ complied with North American TDM consensus (NATDMC) of only collecting trough samples during the treatment course. Vancomycin serum trough concentrations were considered either sub-therapeutic $(70.2 \%, n=158)$ or toxic $(7.6 \%$, $\mathrm{n}=17$ ). In response to the reported toxic vancomycin concentrations, the initial dose was adjusted in $66.7 \%$ of the cases.

Based on SQUH TDM guidelines, $77.4 \%(n=$ 239) of the cases reported both peak and trough serum concentrations. Most of these were within 
the local recommended target concentrations, i.e., $61.5 \%(n=147)$ for peak and $55.2 \%(n=$ 132) for trough.

The dose was adjusted in $66 \%$ of the cases where a toxic serum concentration is reported while it was only adjusted in $30 \%$ of the cases where subtherapeutic serum concentration was reported.

\section{DISCUSSION}

Although vancomycin utilization in SQUH was more for the treatment of infections than prophylactic measures, it was found that only $20.9 \%$ of the prescriptions lie within the range of appropriate use as recommended by HICPAC. This finding is disturbing and may suggest a high degree of vancomycin misuse. Recent studies conducted in Western countries showed that inappropriateness in the use of vancomycin did not exceed $40 \%$ of guidelines recommendations even in the absence of restriction policies [1011]. At SQUH, the prevalence of $\beta$-lactamresistant Gram-positive microorganisms and infections by MRSA, compared to what is reported in most Western countries, is low [12]. Thus, during the study period, only 18 cases were infected with MRSA and all were successfully treated with vancomycin. A similar finding has been reported for Kingdom of Saudi Arabia, a neighboring country [13]. Regardless of this, most of the inappropriateness arose from the continuous use of vancomycin despite receiving negative microbiological culture results for $\quad \beta$-lactam-resistant Gram-positive microorganisms. Possible explanation for this practice is that physicians may have decided to continue empirical use of vancomycin as MRSA might have been undetected in cultures, an indication of lack of awareness of rational use of antibiotics. Another reason for continuing vancomycin as empirical therapy after culture results were available is convenience of use, as its spectrum covers many Gram-positive bacteria. Such trends have been observed in other developing nations as well [14].

Among the other types of inappropriate use of vancomycin were prophylaxis for infections caused by catheter insertions in hemodialysis and peritoneal dialysis, surgical prophylaxis, treatment of Enterococci-related infections and treatment of infections associated with acute chest syndrome in sickle cell disease. VRE is consistently isolated in patients undergoing chronic dialysis especially in those who had previous exposure to vancomycin [15]. Additionally, the use of vancomycin in catheterrelated infections and surgical prophylaxis is indicated only in patients who are allergic to $\beta-$ lactam antibiotics [15]. Thus, the use of vancomycin for the treatment of catheterinsertions related infections is not justified. One of the strategies to limit the spread of VRE is not to use vancomycin in the treatment of Enterococci-related infections [2]. However, this practice was still found in our study. Vancomycin was frequently used for prophylaxis of infections caused by catheter insertions in the nephrology department. In contrast to other hospitals in various countries, the major use of vancomycin was for surgical prophylaxis [6]. Hematology department, however, was the highest prescriber of therapeutic vancomycin, a finding similarly reported elsewhere $[7,10]$. It was utilized for treatment of sepsis and catheter-related infections.

NATDMC recommends the use of trough concentration for monitoring of vancomycin [5]. This is because vancomycin follows a timedependent kinetic profile and nephrotoxicity appears to be associated with specific risk factors such as continuous infusion of vancomycin and concurrent use of loop diuretics and aminoglycosides [16]. The adopted recommended trough vancomycin serum concentration range at SQUH is lower than that currently recommended by NATDMC. Such discrepancy explains why most of the reported serum vancomycin concentrations in both prophylactic and therapeutic groups lie below the therapeutic range. It might also be due to low compliance with the usage recommendations. Recent investigations reveal that for adequate vancomycin therapy and to limit the emergence of resistance, trough vancomycin serum concentration needs to be higher than what was previously thought to be acceptable concentrations [5].

Vancomycin dosage was adjusted more frequently in cases that reported serum concentrations above the therapeutic range than subtherapeutic serum concentrations. Apparently, physicians are concerned more about the toxic effects of vancomycin than subtherapeutic levels. Although toxic vancomycin concentrations might cause adverse effects, subtherapeutic concentrations might lead to failure of therapy and emergence of resistance towards vancomycin [5]. Thus, in both situations, vancomycin dosage change should be considered.

\section{Limitations of the study}

Although we identified several areas that require improvement, such as TDM practice, healthcare 
provider's awareness, and revision of the current vancomycin utilization guidelines, unfortunately, this study did not address the other vancomycinrelated topics. Another major limitation was the nature of the study and the poor documentation in some of the cases. Thus, information such as adverse effects, outcome of vancomycin therapy and appropriate changes made after receiving most of the results were difficult to obtain.

\section{CONCLUSION}

The recorded irrational use of vancomycin indicates deficiencies in utilization practices at the level of study institute and perhaps the entire region. Such practices require further restrictive measures on vancomycin prescribing. Furthermore, the results of TDM review require a re-visit of the recommendation presented in the guidelines to parallel NATDMC guidelines. A further study is required to examine the outcome if the suggested revised recommendations and guidelines are implemented.

\section{Declaration of interest}

None of the authors have any conflict of interests to disclose

\section{REFERENCES}

1. Carmeli $Y$, Samore $M H$, Huskins $C$. The association between antecedent vancomycin treatment and hospital-acquired vancomycin-resistant enterococci: a meta-analysis. Arch Intern Med. 1999; 159: 2461-2468.

2. Rice LB. Emergence of vancomycin-resistant enterococci. Emerg Infect Dis. 2001; 7: 183-187.

3. Kolar M, Urbanek K, Vagnerova I, Koukalova D. The influence of antibiotic use on the occurrence of vancomycin-resistant enterococci. J Clin Pharm Ther. 2006; 31: 67-72.

4. Recommendations for preventing the spread of vancomycin resistance: recommendations of the Hospital Infection Control Practices Advisory
Committee (HICPAC). Am J Infect Control. 1995; 23: 87-94.

5. Rybak M, Lomaestro B, Rotschafer JC, Moellering R, Craig $W$, Billeter M, Dalovisio JR, Levine DP. Therapeutic monitoring of vancomycin in adult patients: a consensus review of the American Society of Health-System Pharmacists, the Infectious Diseases Society of America, and the Society of Infectious Diseases Pharmacists. Am J Health Syst Pharm. 2009; 66: 82-98.

6. Alfandari $S$, Levent $T$, Descamps $D$, Hendricx $S$, Bonenfant $C$, Taines V, Cattoen C, Arimane O, Grandbastien B. Evaluation of glycopeptide use in nine French hospitals. Med Mal Infect. 2010; 40: 232-237.

7. Keyserling $H L$, Sinkowitz-Cochran RL, Harris JM, Levine GL, Siegel JD, Stover BH, Lau SA, Jarvis WR. Vancomycin use in hospitalized pediatric patients. Pediatrics. 2003; 112: e104-111.

8. Roustit M, Francois $P$, Sellier $E$, Roch N, Vittoz JP, Foroni $L$, Stahl JP, Pavese P. Evaluation of glycopeptide prescription and therapeutic drug monitoring at a university hospital. Scand J Infect Dis. 2010; 42: 177-184.

9. Iwamoto $T$, Kagawa $Y$, Kojima M. Clinical efficacy of therapeutic drug monitoring in patients receiving vancomycin. Biol Pharm Bull. 2003; 26: 876-879.

10. Kern WV, De With K, Gonnermann C, Strehl E, Bergner $J$, Hoffken G, Dorje F, Daschner F, Steib-Bauert M, Haber $M$ et al. Update on glycopeptide use in German university hospitals. Infection. 2004; 32: 157-162.

11. Melo DO, Ribeiro E. Vancomycin use in a Brazilian teaching hospital: comparison with the Hospital Infection Controlpractices Advisory Committee Guidelines (HICPAC). Braz J Infect Dis. 2009; 13: 161-164.

12. Richards MJ, Edwards JR, Culver DH, Gaynes RP. Nosocomial infections in combined medicalsurgical intensive care units in the United States. Infect Control Hosp Epidemiol. 2000; 21: 510-515.

13. Al-Tawfiq JA. Incidence and epidemiology of methicillinresistant Staphylococcus aureus infection in a Saudi Arabian Hospital. Infect Control Hosp Epidemiol. 2006; 27: 1137-1139.

14. Isturiz RE, Carbon C. Antibiotic use in developing countries. Infect Control Hosp Epidemiol. 2000; 21: 394-7.

15. Atta MG, Eustace JA, Song $X$, Perl TM, Scheel PJ. Outpatient vancomycin use and vancomycinresistant enterococcal colonization in maintenance dialysis patients. Kidney Int. 2001; 59: 718-724.

16. Catchpole C, Hastings JG. Measuring pre- and post-dose vancomycin levels-time for a change? J Med Microbiol. 1995; 42: 309-311. 